\title{
CETOACIDOSE DIABÉTICA EM CRIANÇAS: PERFIL DE TRATAMENTO EM HOSPITAL UNIVERSITÁRIO
}

\author{
lelma Castro, André Moreno Morcillo, Gil Guerra-Júnior* \\ Trabalho realizado pelo Departamento de Pediatria - Faculdade de Ciências Médicas - UNICAMP, Campinas, SP
}

\author{
*Correspondência: \\ Departamento de Pediatria - \\ FCM - UNICAMP \\ Cidade Universitária \\ Zeferino $\mathrm{Vaz}, \mathrm{s} / \mathrm{n}$ \\ Campinas - São Paulo \\ Caixa Postal 6111 \\ CEP $13083-970$ \\ Tel: (19) 3521-8923 \\ Fax: (19) 3521-8925 \\ gilguer@fcm.unicamp.br
}

\begin{abstract}
RESUMO
Objetivo. Avaliar as características de crianças com cetoacidose diabética (CAD) tratadas no Hospital de Clínicas da UNICAMP.
\end{abstract}

Métodos. Estudo retrospectivo e descritivo de variáveis clínicas e laboratoriais de 74 internações por CAD em 49 pacientes no período de janeiro de 1994 a dezembro de 2003.

Resultados. Doze pacientes tiveram mais de uma internação, 27 eram do sexo feminino e a idade variou de 0,9 a |4,5 anos. O tempo médio de DMI foi de $3 \pm 3,1$ anos, sendo em 20 casos a primeira manifestação do DMI. A CAD foi classificada em grave em $51 \%$ e moderada em 30\%, 17 apresentaram choque e 13 coma na admissão. 0 tempo decorrido para a normalização da glicemia, do pH e do bicarbonato apresentou correlação significativa positiva com o valor inicial. Em apenas três internações foi usado o bicarbonato endovenoso. 0 potássio inicial variou de 3,I a 5,9 $\mathrm{mEq} / \mathrm{l}$, sendo $8 \%$ com valores abaixo de 3,5 e $62 \%$ acima de 4,5. A hipoglicemia ocorreu em 10 internações e 0 edema cerebral com óbito em uma. 0 tempo total de tratamento correlacionou-se significativamente com 0 tempo de fluidoterapia, o local de internação e o tempo para normalização do pH.

Conclusão. Na amostra estudada houve predomínio de pacientes do sexo feminino, abaixo de 10 anos, com manifestação grave da doença, porém com boa evolução e poucas complicações. As internações corresponderam, em sua maioria, a um pequeno número de pacientes.

Unitermos: Acidose. Cetose. Diabetes mellitus. Glicemia. Pediatria. Potássio.

\section{INTRODUÇÃO}

O diabetes mellitus tipo I (DMI) é uma doença crônica grave que acomete milhares de pessoas em todo o mundo, especialmente crianças e adolescentes, necessitando de tratamento intensivo e orientação médica adequada. Após a conclusão do maior estudo envolvendo DMI , o DCCT (Diabetes Controland Complications Trial) concluiu que o tratamento intensivo, regular e disciplinado permite prevenir ou retardar as complicações agudas e crônicas da doença!'.

As principais complicações agudas nos pacientes com DMI são a hipoglicemia e a cetoacidose. A cetoacidose diabética (CAD) é a causa mais comum de hospitalização e morte em crianças diabéticas, e como manifestação inicial do DMI apresenta diferentes incidências conforme a região estudada, variando de 15\% a 67\% na América do Norte e Europa, sendo mais freqüente nos países desenvolvidos ${ }^{2-5}$; como complicação em crianças com DMI diagnosticado previamente, ocorre entre $1 \%$ a $10 \% 5,6$. No Canadá e na Europa, a taxa de hospitalização por CAD em pacientes com DMI ou recém-diagnosticados permanece estável nos últimos 20 anos em 10 para 100.000 crianças, mas a gravidade vem diminuindo ${ }^{7,8}$. Estudo realizado em nosso meio, entre 1989 e 1997, apenas com diabéticos já diagnosticados, mostrou que o número de internações neste período por paciente variou de 0,56 a 0,07/ano, com taxas menores nos últimos anos do estudo? . Recorrência de casos de CAD em um mesmo paciente tem diminuído com a intervenção da equipe multidisciplinar no tratamento crônico do DMI ${ }^{10}$.

As taxas de mortalidade por CAD em estudos com base populacional são relativamente constantes: $0,15 \%$ nos EUA; 0, I8\% a 0,25\% no Canadá e 0,31\% na Inglaterra", sendo a principal causa de mortalidade em pacientes com DMI com idade inferior aos 20 anos $^{12-14}$, tendo o edema cerebral com principal etiologia 15,16 .

Os protocolos para tratamento da CAD são inúmeros, porém a grande maioria inclui a necessidade de internação, preferencialmente em local com equipe treinada para tratamento de urgências, a reposição adequada de fluidos e eletrólitos, a correção da deficiência de insulina, o monitoramento clínico e bioquímico constantes, com o objetivo de restabelecer completamente as alterações metabólicas, evitando-se complicações agudas como edema cerebral, hipoglicemia e hipocalemia ${ }^{10,11,17-19}$.

No Departamento de Pediatria do Hospital de Clínicas $(H C)$ da Universidade Estadual de Campinas (UNICAMP) o tratamento da CAD é realizado tanto no Pronto Socorro, como na Unidade de Terapia Intensiva (UTI) ou na Enfermaria Geral, conforme a gravidade do caso e a disponibilidade de vaga. As equipes dos diversos setores são sempre compostas, além do grupo de enfermagem, de alunos de medicina, 
médicos residentes e médicos assistentes. $O$ tratamento da CAD no Departamento de Pediatria é padronizado e atualizado quando necessário, conforme orientações internacionais ${ }^{10-12}$.

Portanto, o objetivo deste estudo foi conhecer as características das crianças com CAD tratadas no HC - UNICAMP, no período de janeiro de 1994 a dezembro de 2003, em relação à idade, ao sexo, à gravidade da $C A D$, ao local de internação, ao tempo para normalização da glicemia, pH e bicarbonato séricos, ao volume de líquido infundido durante o tratamento, às complicações e ao tempo total do tratamento.

\section{Métodos}

A casuística foi composta de pacientes com CAD (presença de glicemia acima de $200 \mathrm{mg} / \mathrm{dl}, \mathrm{pH}$ abaixo de 7,3 e/ou bicarbonato abaixo de $15 \mathrm{mMol} / /$, acompanhada de glicosúria e cetonúria) ${ }^{10-12}$ internados no Departamento de Pediatria do HC - UNICAMP (Pronto Socorro, UTI ou Enfermaria Geral) no período de janeiro de 1994 a dezembro de 2003. As internações foram levantadas junto ao Serviço de Estatística do HC - UNICAMP utilizando-se como base para a pesquisa o período de tempo, o local de internação e os diagnósticos de DMI e/ou CAD. Foram apenas incluídas no estudo as crianças que preenchiam os critérios diagnósticos de CAD, que tivessem seus prontuários adequadamente preenchidos, com todas as informações necessárias para o estudo, e, no caso de recorrência de internação, que tivessem um período de latência entre uma internação e outra de no mínimo seis meses.

Portanto, de um levantamento inicial de III internações por DMI e/ou CAD em Serviços de Pediatria - HC - UNICAMP, seis foram descartadas por não terem as informações necessárias, 13 por não preencherem os critérios diagnósticos de CAD e 18 por terem recorrência de internação com intervalo menor que seis meses. Restando, ao final, 74 internações por CAD no período analisado (7,4 internações/ano por CAD).

O projeto foi aprovado pelo Comitê de Ética em Pesquisa da Faculdade de Ciências Médicas da UNICAMP.

Trata-se de um estudo retrospectivo e descritivo. Tendo em vista a ocorrência de mais de uma internação para alguns pacientes, denominamos casos, o número de pacientes, e internações, o número total de episódios. A CAD foi classificada ${ }^{11,12,17}$ em leve (pH entre 7,25 e 7,3), moderada ( $\mathrm{pH}$ entre 7,I e 7,24, e/ou bicarbonato entre 10 e I4 $\mathrm{mMol} / \mathrm{l})$ e grave $(\mathrm{pH}<7$, I e/ou bicarbonato $<10 \mathrm{mMol} / \mathrm{l}$ e/ou coma e/ou choque). Todos os pacientes utilizaram insulina regular endovenosa desde a admissão, com dose inicial de 0,I Ul/kg/hora e depois reajustada conforme os valores de glicemia.

As variáveis analisadas foram: local da internação (todos os pacientes deram entrada no HC pelo Pronto-Socorro, porém foi definido como o local de internação o setor do Departamento de Pediatria, onde o paciente permaneceu por maior período durante o tratamento); número de internações por caso; sexo por caso; idade (em anos) na internação; tempo (em anos) de DMI até a internação; presença e local de infecção na admissão; classificaçãa da CAD; presença de coma e/ou choque; glicemia; pH, bicarbonato e potássio séricos na admissão; tempo (em horas) para normalização da glicemia
( $\leq 200 \mathrm{mg} / \mathrm{dl}), \mathrm{pH}(\geq 7,3)$ e bicarbonato ( $\geq 15 \mathrm{mMol} /$ ); hora de início, dose e tempo (em horas) da reposição de potássio; volume de soro utilizado (em $\mathrm{ml} / \mathrm{kg} / \mathrm{h}$ ) nas primeiras seis horas, de seis a 24 horas e o total; tempo total (em horas) de uso do soro endovenoso; uso, hora de início e dose de bicarbonato endovenoso; presença de hipoglicemia (glicemia < $50 \mathrm{mg} / \mathrm{dl}$ ), hora e valor da glicemia; presença de edema cerebral e hora do diagnóstico; evolução para óbito; e tempo total de tratamento (em horas = desde a internação até a suspensão do jejum e da insulina endovenosa).

Para a análise estatística foi utilizado o software SPSS for Windows versão 10.0. Inicialmente, foi realizada uma análise descritiva dos casos, com cálculos de freqüência, média, mediana e desvio-padrão. Também foram utilizados os testes não-paramétricos do Qui quadrado, Exato de Fisher, Correlação por Spearman, Mann-Whitney e Kruskal-Wallis. Para aqueles que tiveram significância neste último, foi realizada uma análise de comparações múltiplas. O nível de significância adotado foi de 5\%.

Para análise da variável volume de soro administrado houve a necessidade de normalização por transformação logarítmica e aplicação da análise de variância e teste T. A análise de regressão linear múltipla foi realizada pelo método "stepwise", utilizando-se como probabilidade de inclusão $p=0,05$ e de exclusão $p=0,10$. A variável resposta tempo (em horas) de tratamento foi também normalizada por transformação logarítmica e as variáveis preditoras testadas no modelo foram o sexo, a idade (em anos) na internação, o tempo (em anos) de DMI, a classificação da CAD (formas moderada e grave), o local de internação (se ProntoSocorro ou UTI), a presença de choque, a presença de coma, o tempo (em horas) de uso de soro (se entre seis e 12 ou acima de 12), o tempo (em horas) de normalização da glicemia (se entre seis e 12 ou acima de 12) e o tempo (em horas) de normalização do pH (se entre seis e 12, entre 13 e 24 ou acima de 24 horas).

\section{Resultados}

As 74 internações corresponderam a 49 casos, sendo que 37 tiveram apenas um episódio de internação e os demais 12 casos com mais de uma internação (dois casos com cinco internações, três com quatro, um com três e seis com duas). Destes 49 casos, 27 (55\%) eram do sexo feminino. A idade em anos na internação variou de 0,9 a I 4,5 (9,9 $\pm 3,5)$ e o tempo em anos de DMI variou de zero a dez ( $3 \pm 3,1)$. Como primeira manifestação do DMI , a CAD ocorreu em 20 casos (41\%) e correspondeu a 27\% das internações. Como fator desencadeante da $C A D$, foi identificado quadro infeccioso na admissão de 15 internações, sendo quatro delas a primeira descompensação do DMI. Os sítios de infecção foram a via aérea superior em cinco internações, a inferior em um, o trato urinário em um, e outros em oito. Não foi observada associação significativa entre o local da infecção e o sexo (Fisher; $p=0,66$ ) e a classificação da CAD, quando analisados casos leves em relação aos moderados mais os graves (Fisher; $p=0,37$ ) e quando apenas moderados em relação aos graves (Fisher; $p=1,00$ ).

Em relação ao local de internação, 38 (5l\%) ocorreram no ProntoSocorro, 21 (28\%) na UTI e as demais I 5 na Enfermaria. Segundo a 
classificação adotada, a CAD foi grave em 38 (5/\%) internações, moderada em $22(30 \%)$ e leve nas demais 14. O choque foi diagnosticado na admissão em 17 (23\%) internações e o coma em 13 (18\%). Na Tabela I encontra-se a distribuição da gravidade da CAD e da presença de coma ou choque segundo o local de internação. Descartados os casos da Enfermaria, observou-se diferença significativa entre a classificação da CAD e o local de internação, sendo os casos mais graves internados na UTI com freqüência maior. Em relação à presença de coma e choque, encontrou-se diferença estatisticamente significativa entre os três locais de internação, com maior freqüência também na UTI.

A Tabela 2 mostra os valores da glicemia, pH e bicarbonato na admissão e o tempo (em horas) para sua normalização.

O tempo decorrido do tratamento para a normalização da glicemia apresentou correlação positiva significativa com seu valor inicial $(p=0,00 \mathrm{I})$, assim como para a normalização do $\mathrm{pH}(\mathrm{p}=0,00 \mathrm{I})$ e do bicarbonato $(p=0,00 I)$.

Em apenas três internações foi utilizado o bicarbonato endovenoso, sendo as três na UTI, com CAD grave e com diagnóstico de choque à admissão. $\mathrm{Na}$ internação com pH 7,08 e bicarbonato $2,8 \mathrm{mMol} / \mathrm{l}$, a dose usada foi de $0,6 \mathrm{mEg} / \mathrm{kg}$ na $15^{\text {a }}$ hora de tratamento; na internação com pH 6,92 e bicarbonato I,9 mMol/, a dose foi de 1,8 mEq/ $/ \mathrm{gg}$ na I I hora; e na internação com pH 6,9 e bicarbonato $2 \mathrm{mMol} / \mathrm{l}$, a dose foi de $2,4 \mathrm{mEq} / \mathrm{kg}$ na $2^{\mathrm{a}}$ hora. O sódio sérico inicial variou de 114 a $151 \mathrm{mEq} / \mathrm{l}(132 \pm 7)$.

Em relação ao potássio, observou-se que a sua concentração sérica inicial variou de 3,1 a $5,9 \mathrm{mEq} / \mathrm{l}(4,6 \pm 0,7)$, sendo seis (8\%) internações com valores iniciais $\leq 3,5 \mathrm{mEq} / \mathrm{l}$ e 46 (62\%) com valores $\geq 4,5 \mathrm{mEq} /$ (limites de normalidade do método utilizado no serviço). O valor da concentração sérica de potássio à admissão não teve associação significativa com a gravidade da cetoacidose $\left(\chi_{(2)}^{2}=0,13, p\right.$ $=0,93)$, com o pH sérico inicial $\left(\chi_{(2)}^{2}=0,94, p=0,62\right)$ ou com a presença ou ausência de choque à admissão $\left(\chi_{(2)}^{2}=0,9, p=0,63\right)$.

A reposição de potássio foi realizada em todas as internações e a dose utilizada foi $2,5 \mathrm{mEq} / 100 \mathrm{ml}$ de solução em oito casos (I | \%); 3 a $4 \mathrm{mEq} / \mathrm{l} 00 \mathrm{ml}$ de solução em quatro (5\%); $5 \mathrm{mEq} / \mathrm{l} 00 \mathrm{ml}$ de solução em 43 (58\%) e 0,3 a 0,5 mEq/kg/hora nos demais I 5 casos. Não houve associação estatisticamente significativa entre a dose utilizada para reposição do potássio e seu valor sérico inicial $\left(\chi_{(2)}^{2}=1,45, p=0,48\right)$, mas foi significativa a associação entre dose de reposição do potássio e a gravidade da $\operatorname{CAD}\left(\chi_{(2)}^{2}=5,2, p=0,07\right)$ e a presença ou não de choque à admissão $\left(\chi_{(2)}^{2}=9,95, p=0,01\right)$. A hora de início da reposição de potássio endovenoso variou de uma a $10(3 \pm 2)$ e o tempo (em horas) de uso foi de uma a 75 (20 \pm 14$)$.

A reposição de fluidos foi feita principalmente com SF 0,9\%, sendo que 0 volume em $\mathrm{ml} / \mathrm{kg} /$ hora nas primeiras seis horas de tratamento variou de cinco a II 0 (40 \pm 22$)$, e da $6^{\mathrm{a}}$ a $24^{\mathrm{a}}$ hora de tratamento, este volume variou de 10 a 200 (57 \pm 36 ). A análise do volume utilizado com relação à gravidade da CAD e à presença de coma ou choque à admissão está descrita na Tabela 3. Observou-se diferença significativa no volume de seis a 24 horas e no volume total em relação à classificação da CAD. O tempo (em horas) de reposição endovenosa de fluidos variou de três a $24(20 \pm 6)$.
Tabela I - Distribuição do local de tratamento das 74 internações por CAD, segundo a gravidade e a presença de choque ou coma à admissão

\begin{tabular}{|c|c|c|c|c|c|c|c|}
\hline & \multicolumn{3}{|c|}{ CAD* } & \multicolumn{2}{|c|}{ Choque** } & \multicolumn{2}{|c|}{ Coma**** } \\
\hline & Leve & Moderada & Grave & Sim & Não & Sim & Não \\
\hline PS & 10 & 13 & 15 & 5 & 36 & 2 & 33 \\
\hline UTI & 0 & 3 & 18 & II & II & 10 & 10 \\
\hline Enfermaria & 4 & 6 & 5 & I & 14 & I & 14 \\
\hline Total & 14 & 22 & 38 & 17 & 57 & 13 & 61 \\
\hline
\end{tabular}

${ }^{*} \chi^{2} c_{(2)}=6,84 ; p=0,03 ; * * \chi_{(2)}^{2}=\left|4,5 ; p=0,001 ; * * * \chi_{(2)}^{2}=\right| 8,3 ; p=0,000 \mid$

\section{Tabela 2 - Dados de glicemia, pH e bicarbonato na admissão das 74 internações por cetoacidose diabética e tempo (em horas) para normalização da glicemia, pH e bicarbonato}

\begin{tabular}{|c|c|c|c|c|}
\hline & \multicolumn{2}{|c|}{ Admissão } & \multicolumn{2}{|c|}{$\begin{array}{c}\text { Tempo(horas) } \\
\text { para normalização }\end{array}$} \\
\hline & $\begin{array}{l}\text { Média } \pm \text { DP } \\
\text { (variação) }\end{array}$ & Mediana & $\begin{array}{l}\text { Média } \pm \text { DP } \\
\text { (variação) }\end{array}$ & Mediana \\
\hline $\begin{array}{l}\text { Glicemia } \\
(\mathrm{mg} / \mathrm{dl})\end{array}$ & $\begin{array}{l}472 \pm 172 \\
(232 \text { a } 976)\end{array}$ & 432 & $\begin{array}{l}6 \pm 5 \\
(\text { I a 24) }\end{array}$ & 4 \\
\hline $\mathrm{pH}$ & $\begin{array}{l}7,12 \pm 0,12 \\
(6,78 \text { a } 7,29)\end{array}$ & 7,13 & $\begin{array}{l}18 \pm 9 \\
(0 a 36)\end{array}$ & 18 \\
\hline $\begin{array}{l}\text { Bicarbonato } \\
\mathrm{mMol} / \mathrm{l}\end{array}$ & $\begin{array}{l}6,7 \pm 3,9 \\
(1,3 \mathrm{a} \mid 4,1)\end{array}$ & 5,9 & $\begin{array}{l}21 \pm 11 \\
(3 \text { a } 36)\end{array}$ & 24 \\
\hline
\end{tabular}

A hipoglicemia esteve presente na evolução de dez internações, sendo que apenas duas destas eram a primeira descompensação do DMI. Os valores da glicemia no momento da hipoglicemia variaram de 14 a $48 \mathrm{mg} / \mathrm{dl}(33 \pm$ 12) e a hora do diagnóstico variou de três a 25 (I3 \pm 6). Não foi observada associação significativa entre a presença de hipoglicemia e sexo $\left(\chi^{2} c_{(1)}=0,25 ; p=0,62\right)$ e a classificação da $\operatorname{CAD}\left(\chi^{2} c_{(2)}=1,07 ; p=0,59\right)$.

Apenas um caso (2\%) ou uma internação (1\%) evoluiu com edema cerebral e óbito. Foi uma menina de 3,4 anos que em 2001 durante a primo-descompensação do DMI internou na UTI em choque e comatosa, com glicemia de $372 \mathrm{mg} / \mathrm{dl}, \mathrm{pH}$ 7,09, bicarbonato 6,I mMol/l, potássio 3,7 mEq/l, sódio $136 \mathrm{mEq} / \mathrm{l}$, fez uso de SF 0,9\% em volume de $30 \mathrm{ml} / \mathrm{kg} /$ hora nas primeiras 6 horas e 1 I $0 \mathrm{ml} / \mathrm{kg} /$ hora após, usou potássio endovenoso na dose de 0,3 a $0,5 \mathrm{mEg} / \mathrm{kg} / \mathrm{hora}$ e apresentou sinais clínicos de edema cerebral com 30 horas de tratamento, irreversível, evoluindo a óbito. As características clínicas e laboratoriais desta paciente não diferem de outras que não apresentaram edema cerebral.

O tempo total (em horas) de tratamento da CAD foi de seis a $80(27 \pm$ 14). Quando o tempo de tratamento foi analisado com relação às variáveis sexo, idade, tempo de DMI, classificação da CAD (formas moderada e grave), local de internação (PS e UTI), presença de choque ou coma à admissão, tempo de fluidoterapia (de seis a 12 horas e mais que 12 horas) e tempo de normalização do $\mathrm{pH}$ e glicemia, somente tempo de fluidoterapia, o PS como local de internação e o tempo de normalização completa do $\mathrm{pH}$ foram significativas. 


\begin{tabular}{|c|c|c|c|c|c|c|c|}
\hline & & \multicolumn{2}{|c|}{$\begin{array}{l}\text { Volume de fluido } \\
1.6 \mathrm{~h}(\mathrm{ml} / \mathrm{kg} / \mathrm{h})^{\prime}\end{array}$} & \multicolumn{2}{|c|}{$\begin{array}{l}\text { Volume de fluido } \\
6.24 \mathrm{~h}(\mathrm{ml} / \mathrm{kg} / \mathrm{h})^{2}\end{array}$} & \multicolumn{2}{|c|}{$\begin{array}{l}\text { Volume total de } \\
\text { fluido }(\mathrm{ml} / \mathrm{kg} / \mathrm{h})^{3}\end{array}$} \\
\hline & & $\mathrm{n}$ & $M \pm D P$ (Variação) & $\mathrm{n}$ & $M \pm D P($ Variação) & $\mathrm{n}$ & $M \pm D P($ Variação) \\
\hline \multirow[t]{3}{*}{ CAD* } & Leve $^{a}$ & 14 & $\begin{array}{c}35,4 \pm 16,2 \\
(15 a 32,5)\end{array}$ & II & $\begin{array}{c}45,9 \pm 33,7 \\
(10 \text { a } 30)\end{array}$ & 14 & $\begin{array}{l}71,4 \pm 44,8 \\
(15 a 57,5)\end{array}$ \\
\hline & Moderada $^{\mathrm{b}}$ & 22 & $\begin{array}{l}40,7 \pm 23,5 \\
(15 a 32,5)\end{array}$ & 19 & $\begin{array}{c}48,7 \pm 43,3 \\
(10 a 45)\end{array}$ & 22 & $\begin{array}{c}82,7 \pm 52,7 \\
(25 \text { a } 75)\end{array}$ \\
\hline & Grave $^{c}$ & 38 & $\begin{array}{l}41,6 \pm 22,5 \\
(10 a 40)\end{array}$ & 37 & $\begin{array}{l}64,1 \pm 32 \\
(15 a 60)\end{array}$ & 38 & $\begin{array}{l}104 \pm 46,5 \\
(20 \text { a I05) }\end{array}$ \\
\hline \multirow[t]{2}{*}{ Choque** } & Sim & 17 & $\begin{array}{c}52,6 \pm 25,9 \\
(15 a 45)\end{array}$ & 17 & $\begin{array}{c}77, I \pm 33,3 \\
(20 \text { a 80) }\end{array}$ & 17 & $\begin{array}{l}129,7 \pm 43,5 \\
(40 \text { a I } 35)\end{array}$ \\
\hline & Não & 57 & $\begin{array}{c}36,4 \pm 18,9 \\
(10 \text { a } 35)\end{array}$ & 50 & $\begin{array}{c}49,8 \pm 34,8 \\
(10 a 45)\end{array}$ & 57 & $\begin{array}{c}80,1 \pm 45,4 \\
(15 a 75)\end{array}$ \\
\hline \multirow[t]{2}{*}{ Coma"** } & Sim & 13 & $\begin{array}{c}46,1 \pm 26,9 \\
(15 \mathrm{a} 40)\end{array}$ & 12 & $\begin{array}{c}75 \pm 35,9 \\
(15 a 65)\end{array}$ & 13 & $\begin{array}{l}115,4 \pm 56,6 \\
(20 \text { a I I } 5 \text { ) }\end{array}$ \\
\hline & Não & 61 & $\begin{array}{c}38,8 \pm 20,4 \\
(10 \text { a } 35)\end{array}$ & 55 & $\begin{array}{c}52,7 \pm 35,3 \\
(10 \text { a } 50)\end{array}$ & 61 & $\begin{array}{c}86,4 \pm 46,6 \\
(15 a 75)\end{array}$ \\
\hline
\end{tabular}

Anova ${ }^{1 *} p=0,78$; teste $T^{1 * *} p=0,69 ;\left.\right|^{1 * * * *} p=0,33$

Anova ${ }^{2 *} p=0,03(a=b ; a=c ; b \neq c) ;$ teste $T^{2 * *} p=0,19 ; 2^{2 * * *} p=0,26$

Anova ${ }^{3 *} p=0,04(a=b ; b=c ; a \neq c) ;$ teste $T^{3^{3 *}} p=0,12 ;{ }^{3 * n} p=0,57$

\begin{tabular}{c|c}
\hline$R^{2}$ ajustado & Modelo \\
\hline 0,489 & $T=1,05|+0,44|(T S 2)-0,|I 5(P S)+0,20|(T S \mid)+0, \mid 53(T p H)$ \\
\hline
\end{tabular}

$T=$ tempo (em horas) de tratamento;

TS2 = tempo de soro $(0$ = abaixo de 12 horas, $\mid$ = acima de 12 horas $) ;$

$\operatorname{PS}(0=$ outro local; $I=P S)$;

$\mathrm{TS} \mid=$ tempo de soro $(0$ = abaixo de 6 e acima de 12 horas; $\mid$ = entre 6 e 12 horas);

$\underline{\mathrm{TpH}}=$ tempo de normalização do pH ( 0 = abaixo de 24 horas; I = acima de 24 horas)

\section{Discussão}

A incidência do DMI em todo o mundo varia de 0,1 a 36,8 / 100.000/ano e no Brasil, dados de São Paulo, mostram valor próximo a 8/100.000/ano $0^{20,21}$. A despeito de todos os conhecimentos e estratégias terapêuticas adquiridas nas últimas décadas tanto para o tratamento do DMI como para a CAD, não há diminuição das taxas de mortalidade na CAD, que giram em torno de $1 \%$ a $2 \%$ desde $1970^{10-16}$. Estes dados mostram a importância de se conhecer a população de DMI que apresenta CAD no sentido de encontrar características que possam auxiliar na sua prevenção ou na diminuiç̧ão da sua mortalidade.

As 74 internações por CAD avaliadas no presente estudo ocorreram em 49 pacientes com DMI, sendo que 37 destes $(75,5 \%)$ tiveram uma única internação no período de 10 anos do estudo. As outras 37 internações (50\%) foram relativas aos demais 12 pacientes. Portanto, alguns pacientes são responsáveis pela metade das internações, apontando para a necessidade de uma intervenção maior e mais enfática no tratamento ambulatorial do DMI, especialmente na educação, visto que, segundo a literatura, em cerca de $75 \%$ dos casos a CAD está associada ao não uso ou uso inadequado da insulina ${ }^{1,12,22}$. Os pais ou responsáveis devem estar estreitamente envolvidos no tratamento, visto que a média de idade dos pacientes internados foi de 9,9 anos e variou de 10 meses a 14 anos e 6 meses. Um outro fator precipitante importante da CAD é a infecção (20 a 25\%), sendo a pneumonia e a infecção do trato urinário os tipos mais comuns ${ }^{17,19}$. Neste estudo, a infecção esteve presente como fator desencadeante, único ou associado a outro, em $20 \%$ dos casos; porém as infecções de vias aéreas inferiores e trato urinário foram as menos freqüentes, sendo a infecção da via aérea superior a mais comum, mostrando que mesmo infecções leves são capazes de precipitar o aparecimento da CAD em pacientes com controle crônico irregular do DMI.

Em 27\% das internações ou 4I\% dos casos, a CAD foi a primeira manifestação do DMI, coincidindo com dados mundiais, que mostram uma variação entre 15 e 67\% 2.5 .

Quanto à gravidade da CAD à admissão, $51 \%$ foram consideradas graves, 30\% moderadas e apenas 19\% leves. Apesar de não haver dados relatando freqüência de apresentação de cada um destes grupos em outros serviços, pode-se inferir que, mais uma vez há aqui refletido o controle domiciliar ruim dos pacientes, associado muitas vezes à demora da busca pelo serviço de saúde ou muitas vezes o atendimento inadequado em unidades básicas de saúde que antecedem o encaminhamento ao serviço terciário de referência.

A maior parte dos casos foi internada no PS, onde eram inicialmente avaliados e na UTI. Quando avaliada a gravidade com relação ao local de internação, tem-se que, conforme previsto, os casos mais graves foram internados na UTI com maior freqüência. O fato de alguns casos graves terem sido encaminhados à enfermaria ou permanecido no PS, pode-se inferir que ocorreu, provavelmente pela não disponibilidade de vagas, visto que se trata de serviço público e terciário, com grande demanda de pacientes graves. Os casos mais leves foram encaminhados à enfermaria, provavelmente por ser o setor onde 0 monitoramento constante, clínico e laboratorial do paciente, nem sempre é possível. 
O presente estudo também demonstrou que o tempo decorrido de tratamento foi diretamente proporcional às alterações laboratoriais iniciais, evidenciando a importância do monitoramento glicêmico domiciliar e do reconhecimento precoce dos familiares da hiperglicemia mantida e de seus sintomas, diminuindo assim o tempo de tratamento, os riscos e os gastos. O último consenso para o tratamento do DMI recomenda que os casos de hiperglicemia com cetose e sem acidose devem ser tratados domiciliarmente pelo telefone, com ajuda de equipe treinada ou na unidade básica de saúde mais próxima do domićlio"1,12,23.

O uso de bicarbonato no tratamento da CAD em crianças não é recomendado, visto que vários estudos já demonstraram ausência de benefícios e aumento dos riscos ${ }^{11,12,16,24,25}$. Porém, há falta de estudos comparando seu uso em pacientes muito graves, com acidemia grave e mantida a despeito do tratamento adequado da CAD. No presente estudo, o bicarbonato endovenoso foi utilizado em apenas três internações (0,04\%), todas na UTI, sendo que seu uso talvez seja justificado pelo fato de serem todas de pacientes graves, com acidemia e bicarbonato sérico bastante baixos e, em duas delas, a infusão de bicarbonato ocorreu após mais de dez horas de tratamento adequado, devido a não melhora do pH e do bicarbonato com o tratamento habitual da CAD.

Com relação ao potássio, este estudo comprovou que na maioria das internações o potássio sérico inicial estava normal ou elevado, com apenas $8 \%$ apresentando hipopotassemia à admissão. A hiper ou normo potassemia na admissão é esperada pela própria fisiopatologia da CAD. Porém, sabe-se que sempre há um déficit do "pool" global de potássio a não ser que o paciente encontre-se em insuficiência renal. Por esse motivo, recomenda-se a reposição de potássio em todos os casos de CAD, como ocorreu neste estudo. Porém, a dose utilizada para a reposição não apresentou associação significativa com valor sérico inicial de potássio. Talvez, outros valores de potássio durante o tratamento tenham sido levados em consideração na escolha da dose de reposição. A hora de início da reposição variou de uma a 10 horas, evidenciando que o início tardio ocorreu em alguns casos, sendo que a literatura recomenda a reposição assim que se inicie a fluidoterapia de manutenção, desde que não haja dúvidas quanto ao funcionamento normal do sistema renal|9,22,25. Apesar disso, a dose de reposição do potássio apresentou associação significativa em relação à gravidade da $C A D$, à presença ou não de choque e ao local de internação, sendo utilizadas doses maiores nos casos mais graves de CAD, nos quais havia presença de instabilidade hemodinâmica a nos casos internados em UTI, conforme esperado.

Quanto à fluidoterapia, a solução salina 0,9\% foi predominantemente utilizada, sendo que nas primeiras seis horas o volume variou de 5 a $110 \mathrm{ml} / \mathrm{kg} / \mathrm{h}$, da $6^{\text {a }}$ à $24^{\mathrm{a}}$ hora variou de 10 a $200 \mathrm{ml} / \mathrm{kg} / \mathrm{h}$. Quando analisado o volume de fluido utilizado com relação à gravidade da CAD, houve diferença significativa do volume infundido de seis a 24 horas com relação à gravidade e do volume total com relação à gravidade e local de internação. Esse resultado estaria de acordo com previsto, porém caso tivesse sido encontrada diferença significativa no volume infundido com relação à presença de coma e choque, isto é, infusão mais cautelosa no primeiro e mais vigorosa na presença do último. $\mathrm{O}$ fato de não haver associação significativa do volume utilizado nas primeiras seis horas com a gravidade da CAD evidencia um possível excesso na oferta hídrica, a qual deveria ser limitada em $40 \mathrm{ml} / \mathrm{kg}$ nas primeiras quatro horas, exceto na presença de choque. Apesar de constar como recomendação na maioria dos protocolos, a associação entre volume infundido e risco de edema cerebral ainda permanece

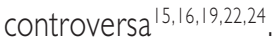

A hipoglicemia ocorreu em 13,5\% das internações e não apresentou associação significativa com o sexo ou com a gravidade da CAD. Sabe-se que antes do advento do uso de infusão contínua de baixas doses de insulina, a hipoglicemia que era uma complicação freqüente durante o tratamento da CAD, com incidências acima de $25 \%$, tornou-se menos comum ${ }^{19,25}$.

O único caso deste estudo que apresentou edema cerebral com manifestação clínica e evolução para óbito não tinha características clínicas ou laboratoriais diferentes dos outros pacientes. Portanto, a incidência de edema cerebral neste estudo foi de $2 \%$ dos casos e $1 \%$ das internações, sendo que incidência descrita em literatura varia de 0,2 a $2 \%{ }^{15,16,22,26}$.

O tempo total de tratamento neste estudo apresentou correlação com o tempo de fluidoterapia, com o local de internação e com o tempo de normalização do pH. Estes dados mostram indiretamente uma relação com a gravidade do caso.

\section{Conclusão}

Apesar de toda a heterogeneidade de pessoas e locais envolvidos na realização e descrição do tratamento destes casos de CAD, os dados encontrados nos prontuários e analisados são relativamente homogêneos, o que de certa forma denotam a importância da padronização e o bom censo dos responsáveis por este tratamento.

Portanto, levando-se em conta os dados deste grupo de internações por $C A D$, pode-se concluir que esta doença predomina em um grupo restrito de crianças, freqüentemente do sexo feminino, com idade abaixo de 10 anos, com manifestação grave da doença, porém com evolução favorável e com poucas complicações.

\section{Agradecimentos}

A todos os membros das equipes de saúde do Pronto-Socorro, Enfermaria e Unidade de Terapia Intensiva do Departamento de Pediatria do Hospital de Clínicas e Faculdade de Ciências Médicas da UNICAMP responsáveis pelo atendimento destes pacientes e o preenchimento correto das informações nos prontuários.

\section{Conflito de interesse: não há}

\section{SUMMARY}

\section{Diabetic KetoAcidosis IN CHILDREN: tREATMent PROFILE AT A UNIVERSITY HOSPITAL}

OBIECTNE. To evaluate the profile of children with diabeticketoacidosis (DKA) treated at the UNICAMP "Hospital de Clínicas".

Methods. Retrospective and descriptive study of clinical and laboratory variables of 74 admissions related to 49 patients bearers of DKA, between January 1994 and December 2003. 
RESULTS. Twelve patients were admitted more than once, 27 were females and ages ranged from 0.9 to 14.5 years. The mean time from DMI diagnosis to admission was $3 \pm 3.1$ years and 20 cases presented with DKA at the onset of DMI. DKA was severe in $51 \%$ and moderate in $30 \%$, at admission 17 patients presented with shock and 13 coma. The time needed for normalization of glycemia, pH, and bicarbonate had a significant positive correlation with the initial value. Intravenous bicarbonate was administered in only 3 admissions. The initial potassium value ranged from 3.1 to $5.9 \mathrm{mEq} /$; in $8 \%$ the value was $/$ wer than 3.5 and in $62 \%$ higher than 4.5. Hypoglycemia occurred in 10 admissions and cerebral edema and death in one. The total time of treatment had a significant correlation with the time of fluid replacement, place of admission and time needed for $\mathrm{pH}$ normalization.

CONCLUSION. The analyzed sample showed a predominance offemale patients, less than ten years of age with a severe manifestation of the disease. However, evolution was good with few complications. Most of the admissions refer to a small number of patients. [Rev Assoc Med Bras 2008; 54(6): 548-53]

KEY wORDs: Acidosis. Blood glucose. Diabetes mellitus. Ketosis. Pediatrics. Potassium.

\section{REFERÊNCIAS}

I. The Diabetes Control and Complications Trial Research Group. The effect of intensive treatment of diabetes on the development and progression of long-term complications in insulin-dependent diabetes mellitus. N Engl J Med 1993;329:977-85.

2. Pinkey JH, Bingley PJ, Sawtell PA, Dunger DB, Gale EA. Presentation and progress of childhood diabetes mellitus: a prospective population-based study. The Bart's-Oxford Study Group. Diabetologia 1994:37:70-4.

3. Levy-Marchal C, Papoz L, de Beaufort C, Doutreix J, Froment V, Voirin J et al. Clinical and laboratory features of type I diabetic children at the time of diagnosis. Diabet Med 1992;9:279-84.

4. Komulainen J, Lounamaaa R, Knip M, Kaprio EA, Akerblom HK. Ketoacidosis at the diagnosis of type I (insulin dependent) diabetes mellitus is related to poor residual beta cell function. Childhood Diabetes in Finland Study Group. Arch Dis Child 1996;75:4 I 0-5.

5. Smith CP, Firth D, Bennett S, Howard C, Chisholm P. Ketoacidosis occurring in newly diagnosed and established diabetic children. Acta Paediatr 1998;87:537-4|.

6. Morris AD, Boyle DI, McMahon AD, Greene SA, MacDonald TM, Newton RW. Adherence to insulin treatment, glycaemic control, and ketoacidosis in insulin-dependent diabetes mellitus. Diabetes Audit and Research in Tayside Scotland. Medicines Monitoring Unit. Lancet 1997;350: 1505-10

7. Curtis JR, To T, Muirhead S, Cummings E, Daneman D. Recent trends in hospitalization for diabetic ketoacidosis in Ontario children. Diabetes Care 2002;25: 159|-6.

8. Hirasing RA, Reeser HM, de Groot RR, Ruwaard D, van Buuren S, Verloove-Vanhorick SP. Trend in hospital admissions among children aged 0-19 years with type I diabetes in the Netherlands. Diabetes Care 1996; | 9:431-4.
9. Lemos-Marini SHV, Lima MC, Guerra-Júnior G, Paulino MFVM, Minicucci WJ. A importância dos controles domiciliares na redução de internação em portadores de diabetes mellitus tipo I. Arq Bras Endocrinol Metab 2000;44:215-9.

10. Rosenbloom AL, Hanas R. Diabetic ketoacidosis (DKA): treatment guidelines. Clin Pediatr 1996;35:26I-6.

II. Dunger DB, Sperling MA, Acerini CL, Bohn DJ, Daeman D, Sanne TP et al. European Society for Paediatric Endocrinology and Lawson Wilkins Pediatric Endocrine Society. Consensus statement on diabetic ketoacidosis in children and adolescents. Pediatrics 2004; I 13:133-40.

12. Wolfsdort J, Glasser H, sperling MA, American Diabetes Association. Diabetic ketoacidosis in infants, children and adolescents: a consensus statement from the American Diabetes association. Diabetes Care 2005;28:2384-7.

13. Dahlquist G, Källén B. Mortality in childhood-onset type I diabetes. A population-based study. Diabetes Care 2005;28:2384-7.

14. Melo MS, Lolio CA, Lucena MAF, Kirzner CF, Martins SM, Barros MNDS Causas múltiplas de morte em diabéticos no município de Recife, 1987. Rev Saúde Públ 1991;25:435-42.

15. Edge JA, Hawkins MM, Winter DL, Dunger DB. The risk and outcome of cerebral oedema developing during diabetic ketoacidosis. Arch Dis Child 2001;85:16-22.

16. Glaser N, Barnett P, McCaslin I, Nelson D, Trainor J, Louie J et al. Risk factors for cerebral edema in children with diabetic ketoacidosis. The Pediatric Emergence Medicine Collaborative Research Committee of American Academy of Pediatrics. N Engl J Med 200 I;344:264-9.

17. Trachtenbarg DE. Diabetic ketoacidosis. Am Fam Physician 2005:71: 1705-14.

18. Ilag LL, Kronik S, Ernst RD, Grondin L, Alaniz C, Liu L et al. Impact of a critical pathway on impatient management of diabetic ketoacidosis. Diabetes Res Clin Pract 2003;62:23-32.

19. Kitabchi AE, Umpierrez GE, Murphy MB, Barrett EJ, Kreisberg RA, Malone Jl et al. Hyperglycemis crises in diabetes. Diabetes Care 2004;27 (suppl I):S94-102.

20. Karvonen M, Viik-Kajander M, Moltchanova E, Libman I, LaPorte R, Tuomilehto J. Incidence of childhood type I diabetes worldwide. Diabetes Care 2000;23:1516-26.

21. Ferreira SRG, Franco LJ, Vivolo MA, Negrato CA, Simões ACP, Ventureli CR. Population-based incidence of IDDM in the state of São Paulo, Brazil. Diabetes Care 1993; 16:701-4.

22. White NH. Management of diabetic ketoacidosis. Rev Endocr Metabol Disord 2003; 4:343-53.

23. Viallon A, Zeni F, Lafond P, Venet C, Tardy B, Page $Y$ et al. Does bicarbonate therapy improve the management of severe diabetic ketoacidosis? Crit Care Med 1999;27:2690-3.

24. Piva JP, Czepielewski M, Garcia PCR, Machado D. Current perspectives for treating children with diabetic ketoacidosis. J Pediatr (Rio J) 2007;83(5 suppl):SI I 9-27.

25. Chiasson J, Nahla A, Bélanger R, Bertrand S, Beauregard H, Èkoé J et al. Diagnosis and treatment of diabetic ketoacidosis and hyperglycemic hyperosmolar state. Can Med Am J 2003;168:859-66.

26. Muir A. Cerebral edema in diabetic ketoacidosis: a look beyond rehydration. J Clin Endocrinol Metab 1985;85:509-13.

Artigo recebido: 18/08/07

Aceito para publicação: 12/06/08 